\title{
Intricate Physics of Coherent Electron Beam/Oxide Materials Interaction Revealed by 4D Inline Holography_Electron Ptychography
}

\author{
Huolin L. Xin ${ }^{1}$, Xiaoyan Zhong ${ }^{2}$, Jie Lin $^{2}$, Zhenyu Liao ${ }^{2}$, Jing Zhu ${ }^{2}$ and Xiaojing Huang ${ }^{3}$ \\ 1. Center for Functional Nanomaterials, Brookhaven National Laboratory, Upton, NY, USA \\ 2. National Center for Electron Microscopy in Beijing, Key Laboratory of Advanced Materials (MOE), \\ State Key Laboratory of New Ceramics and Fine Processing, School of Materials Science and \\ Engineering, Tsinghua University, Beijing, China. \\ 3. National Synchrotron Light Source II, Brookhaven National Laboratory, Upton, New York, United \\ States.
}

Wave-front engineering in electron microscopy has attracted much attention due to its promises for detecting magnetism at the atomic scale. This approach has been realized by passing electron plane waves through a pre-designed phase plate to achieve a desired magnitude/phase pattern in the far field. For example, by passing electrons through a pitch-fork aperture, a coherent electron beam can be splitted into a series of sub-beams that carry discrete orbital angular momenta, or topological charge. This type of electron beams is also called an electron vortex beam because its possibility current revolves around the propagating axis. With the current fabrication technology, condenser hologram apertures can be made to create atomic-scale electron vortex beams. Much hope in this field is to utilize these small vortex beams to detect localized dichroism signals with atomic resolution. Unfortunately, the orbital angular momentum of an electron beam cannot couple efficiently with the spin dimension of the core-binding electrons. The only way to detect magnetic signals in the inelastic channel is to measure dichroism signals resulted from spin-orbit coupling. Nevertheless, this approach has not been as promising as expected primarily due to the limitation of broken rotational symmetry when the electron beam is moved off the atomic columns (both $\Delta m= \pm 1$ and 0 are allowed transitions in electron inelastic scattering with equal probability). Even when the vortex beam is exactly on an atomic column, the dichroism signal is much weaker than those in traditional EMCD. This has been an unsurmountable barrier since the initial discovery of an electron vortex beam in 2010. [1,2]

To overcome this limitation, Verbeeck and his colleagues offered a solution in their work published in 2010. [2] Basically as opposed to using the pitch-fork aperture as a way of generating vortex beams above the sample, they used the pitch-fork aperture as a post-specimen chiral analyser. Most works that followed Verbeeck et al (2010) only picked up the idea of shaping wave-fronts using a patterned phase plate, but the idea of chiral analyser has largely been overlooked by the field because this part of the results is very difficult to reproduce. The difficulty stems from the following. First, for the chiral analyser to work, the demagnified size of the analyser needs to match the atomic dimension. Given the objective aperture are at least a few micrometers in size, this is not the case if the microscope is operated in the normal conditions. Second, the separation between the side bands are only a few micro-rad in angle. This makes the acquisition of EELS signal from the correct angular regions very difficult. Therefore, to improve the pitch-fork chiral analyser, we need to reduce the dimension of the pitch-fork aperture and increase the angular separation between the side bands.

In this talk, I will give an overview of the field and discuss how to create atomic-scale chiral analyzer by exploiting oxide materials and how to improve the side-band angular separation by three orders of magnitude. I will show how these spatial features could give rise to exotic vortex orders when they 
interact with a high energy electron beam. To probe this interaction, we used the electron ptychographic technique, recovered the missing phase information in the diffraction plane and revealed the topological phase vortices in the diffracted beams [3].

\section{References:}

[1] Uchida, M. \& Tonomura, A. Nature 464 (2010) p. 737.

[2] J. Verbeeck, H. Tian and P. Schattschneider, Nature 467 (2010), p. 301.

[3] H.L.X. acknowledges support from Center for Functional Nanomaterials, Brookhaven National Laboratory, which is supported by the U.S. Department of Energy, Office of Basic Energy Sciences, under Contract No. DE-AC02-98CH10886.

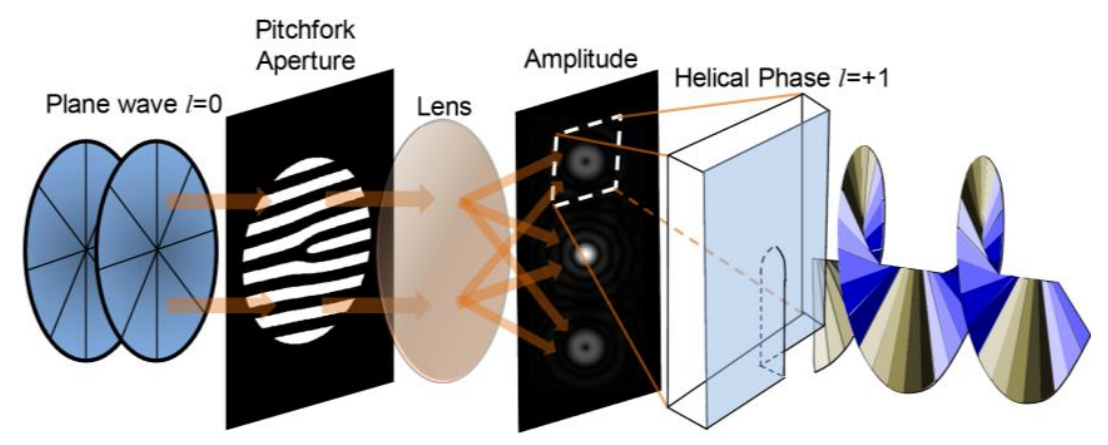

Figure 1. Electron vortex beams. Schematic showing a plane wave of electrons being converted to a vortex beam by a pitchfork hologram mask placed in the front focal plane of the electron lens.

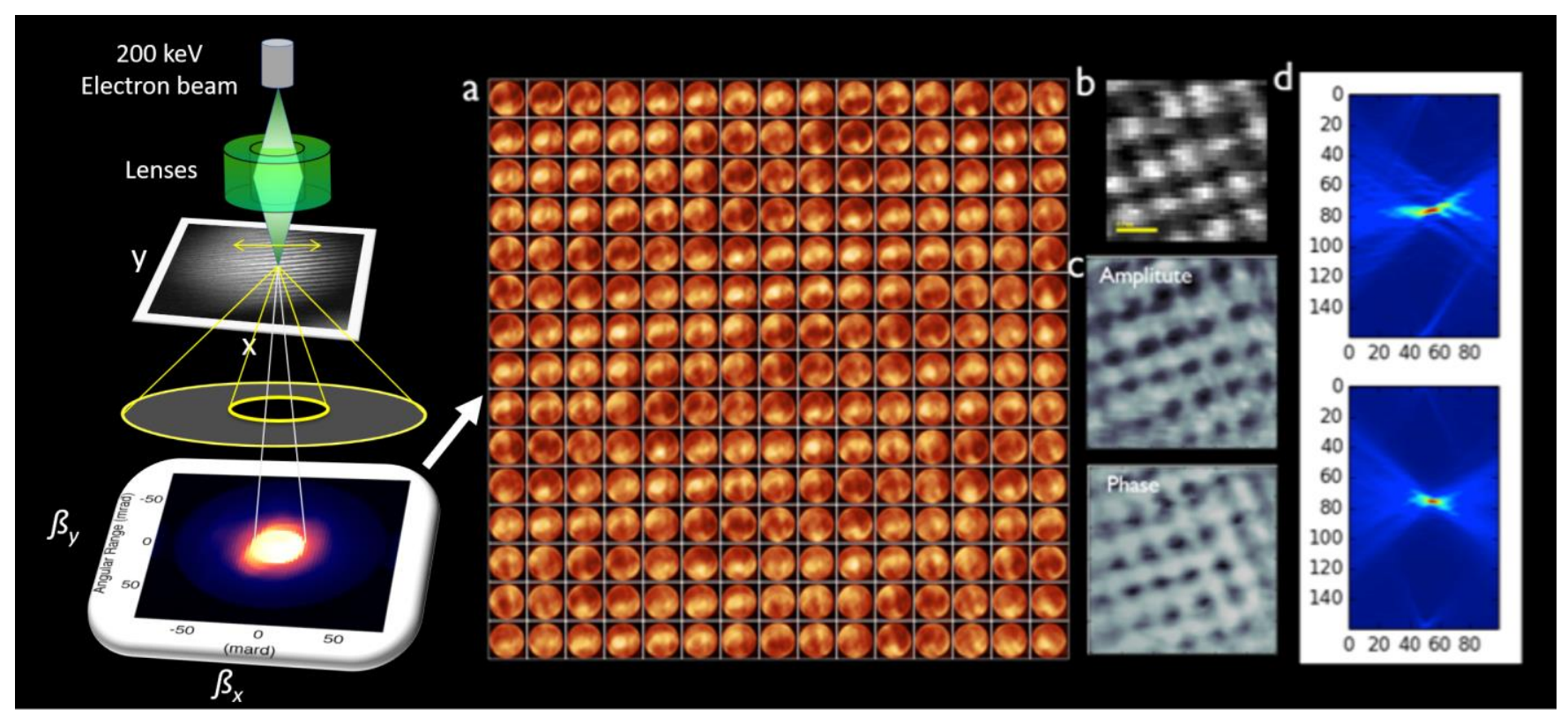

Figure 2. In-focus position-dependent coherent CBED mapping with sufficient overlapping orders allows for ptychographic reconstruction of atomic lattices. (a) The position-dependent CBED patterns. (b) The ADF-STEM map. (c) The ptychographic reconstruction of the object function. (d) The reconstructed probe wave front viewed in $\mathrm{x}-\mathrm{z}$ and $\mathrm{y}-\mathrm{z}$ cross sections. 